INVESTIGACIÓN

http://doi.org/10.15198/seeci.2019.50.27-42

Recibido: 15/02/2019 --- Aceptado: 17/09/2019 --- Publicado: 15/11/2019

\title{
REPRESENTACIONES CONCEPTUALES MULTIMEDIA DE LOS ÁMBITOS DE ACTUACIÓN EN EDUCACIÓN SOCIAL ELABORADOS POR ESTUDIANTES UNIVERSITARIOS (2015-2018)
}

\section{MULTIMEDIA CONCEPTUAL REPRESENTATIONS OF WORK AREAS IN SOCIAL EDUCATION PREPARED BY UNIVERSITY STUDENTS (2015-2018)}

Eloy López Meneses ${ }^{1}$ : Universidad Pablo de Olavide. España. elopmen@upo.es

Esteban Vázquez Cano²: Universidad Nacional de Educación a Distancia. España. evazquez@edu.uned.es

Esther Fernández Márquez³: Universidad Pablo de Olavide. España. efermar2@upo.es

Jesús López Belmonte4: Universidad Internacional de Valencia. España. jesus.lopezb@campusviu.es

La presente investigación surge de la directriz marcada por el proyecto Innovación docente 2.0 con Tecnologías de la Información y la Comunicación en el Espacio Europeo de Educación Superior, desarrollado en el marco de los Proyectos de Innovación y Desarrollo Docente de la Universidad Pablo de Olavide (UPO), financiado por el Vicerrectorado de Docencia y Convergencia Europea de dicha Universidad.

\footnotetext{
${ }^{1}$ Eloy López Meneses: Profesor titular de la Universidad Pablo de Olavide. Director del grupo de investigación Eduinnovagogía (HUM-971).

elopmen@upo.es

${ }^{2}$ Esteban Vázquez Cano: Profesor de la Facultad de Educación de la UNED. Miembro del grupo de investigación (RADTE) Estrategias Metodológicas para la Construcción de una Red a Distancia de Tecnología Educativa (RADTE).

evazquez@edu.uned.es

${ }^{3}$ Esther Fernández Márquez: Profesora de la Universidad Pablo de Olavide. Doctora en Educación y Comunicación en la Universidad de Málaga. Miembro del grupo de investigación Eduinnovagogía (HUM-971). Línea de investigación: Formación del profesorado en competencias digitales e innovación en tecnologías emergentes.

efermar2@upo.es

"Jesús López Belmonte: Doctor en Ciencias de la Educación. Profesor de la Universidad Internacional de Valencia. Miembro del Grupo de investigación AREA (HUM-672). Máster en tecnología educativa y competencias digitales.

jesus.lopezb@campusviu.es
} 
López Meneses, E.; Vázquez Cano, E.; Fernández Márquez, E. y López Belmonte, J.

Representaciones conceptuales multimedia de los ámbitos de actuación en educación social elaborados por estudiantes universitarios (2015-2018)

\section{RESUMEN}

El presente estudio emana del proyecto Innovación docente 2.0 con Tecnologías de la Información y la Comunicación en el Espacio Europeo de Educación Superior, desarrollado en el marco de los Proyectos de Innovación y Desarrollo Docente de la Universidad Pablo de Olavide (UPO), financiado por el Vicerrectorado de Docencia y Convergencia Europea de dicha Universidad. La investigación describe un estudio diacrónico sobre las concepciones acerca de los ámbitos propios de la Educación Social mediante la elaboración de mapas conceptuales multimedia. El desarrollo de la misma plantea un carácter innovador y se desarrolla con 266 estudiantes pertenecientes a los cursos de Grado de Educación Social y Doble Grado de Educación Social y Trabajo Social de la Universidad Pablo de Olavide, durante los cursos académicos 2015/16, 2016/17 y 2017/2018. A través de una metodología cualitativa, mediante técnicas de etnografía virtual y cuantitativa, se analizaron los ámbitos de intervención en Educación Social y Trabajo Social con los siguientes resultados: personas con discapacidad y salud mental $(15,37 \%)$, drogodependencia $(14,70 \%)$, tercera edad $(14,03 \%)$, adultos $(11,25 \%)$, infancia $(9,47 \%)$, inmigración $(8,35 \%)$, animación sociocultural $(7,57 \%)$, violencia de género $(6,12 \%)$ y adolescencia (4,34\%).

PALABRAS CLAVE: educación superior - aprendizaje en red - estrategia de enseñanza virtual - mapa conceptual multimedia - innovación educativa - formación para la docencia universitaria - recursos en línea.

\section{ABSTRACT}

The present study arises from the Teaching Innovation 2.0 project with Information and Communication Technologies in the European Higher Education Area, developed within the framework of the Innovation and Teaching Development Projects of the Pablo de Olavide University (UPO), financed by the Vice-rectorate of Teaching and European Convergence. The research describes a diachronic study on the conceptions of the specific fields of work in Social Education through the elaboration of multimedia conceptual maps. An innovative planning is carried out and it is developed with 266 students from the courses of Degree of Social Education and Double Degree of Social Education and Social Work of the Pablo de Olavide University, during the academic years 2015/16, 2016/17 and 2017/2018. Through a qualitative methodology, with virtual and quantitative ethnography techniques, the intervention areas in Social Education and Social Work were analyzed with the following results: people with disabilities and mental health (15.37\%), drug dependence $(14.70 \%)$, third age $(14.03 \%)$, adults $(11.25 \%)$, childhood $(9.47 \%)$, immigration $(8.35 \%)$, sociocultural animation $(7.57 \%)$, gender violence $(6.12 \%)$ and adolescence $(4.34 \%)$.

KEY WORDS: higher education - online learning - virtual teaching strategy multimedia conceptual map - educational innovation - training for university teaching - on-line resources. 
López Meneses, E.; Vázquez Cano, E.; Fernández Márquez, E. y López Belmonte, J. Representaciones conceptuales multimedia de los ámbitos de actuación en educación social elaborados por estudiantes universitarios (2015-2018)

\section{REPRESENTAÇÕES CONCEITUAIS MULTIMIDIA DOS ÂMBITOS DE ATUAÇÃO EM EDUCAÇÃO SOCIAL ELABORADOS POR ESTUDANTES UNIVERSITÁRIOS (2015-2018)}

\section{RESUME}

O presente estudo emana do projeto Innovacion docente 2.0 con Tecnologias de la Informacion y la Comunicacion en el Espacio Europeo de Educacion Superior, desenvolvido no marco dos Projetos de Inovação e Desenvolvimento Docente da Universidade Pablo de Olavide (U.P.O.), financiado pelo Vice-Reitorado de Docência e Convergência Europeia desta universidade. A investigação descreve um estudo diacrônico sobre as concepções sobre os âmbitos próprios da Educação Social mediante a elaboração de mapas conceptuais multimídia. O desenvolvimento da mesma propõe um caráter inovador e se desenvolve com 266 estudantes pertencentes aos cursos universitários de Educação Social e Trabalho Social da U.P.O., durante os cursos acadêmicos 2015/16, 2016/17 e 2017/18. Através de uma metodologia qualitativa, mediante técnicas de etnografia virtual e quantitativa, se analisaram os âmbitos de intervenção em Educação Social e Trabalho Social com os seguintes resultados: pessoas com incapacidade e saúde mental $(15,37 \%)$, toxicômanos $(14,70 \%)$, terceira idade $(14,03 \%)$, adultos $(11,25 \%)$, infância $(9,47 \%)$, imigração $(8,35 \%)$, animação sociocultural $(7,57 \%)$, violência de gênero $(6,12 \%)$ e adolescência $(4,34 \%)$.

PALAVRAS CHAVE: educação superior - aprendizagem em rede - estratégia de ensino virtual - mapa conceitual multimídia - inovação educativa - formação para docência universitária - recursos online.

\section{Cómo citar el artículo:}

López Meneses, E.; Vázquez Cano, E.; Fernández Márquez, E. y López Belmonte, J. (2019). Representaciones conceptuales multimedia de los ámbitos de actuación en educación social elaborados por estudiantes universitarios (2015-2018). [Multimedia conceptual representations of work areas in social education prepared by university students (2015-2018)]. Revista de Comunicación de la SEECI, (50), 27-42. doi: http://doi.org/10.15198/seeci.2019.50.27-42

Recuperado de http://www.seeci.net/revista/index.php/seeci/article/view/570

\section{INTRODUCCIÓN}

El concepto de educación requiere una reconceptualización, por parte de docentes y estudiantes, de cara a los desafíos de la revolución digital en términos comunicativos, que impactan directamente en los procesos educativos (RodríguezGarcía, 2017). En este sentido, en el contexto universitario es necesario un cambio metodológico y evaluador orientado que potencie la formación integral de los estudiantes a través del desarrollo de sus competencias sociales, intelectuales y tecnológicas (Aguaded, López-Meneses \& Jaén, 2013). En este sentido, los mapas conceptuales multimedia pueden ser elementos curriculares relevantes en los 
procesos de innovación metodológica y en el desarrollo de estrategias metacognitivas (Fernández-Márquez, Vázquez-Cano \& López Meneses, 2016; López Meneses, Vázquez-Cano \& Jaén Martínez, 2017).

Novak y Cañas (2008) indican que un mapa conceptual es una herramienta de organización jerárquica de conceptos (regularidades percibidas en sucesos u objetos, o registros de sucesos u objetos, designadas mediante etiquetas) y de determinación de relaciones entre estos conceptos, que constituyen proposiciones o unidades semánticas coherentes (sentencias que permiten una descripción autosuficiente de un objeto o suceso contenido en el universo de estudio). Y se utilizan para la generación, visualización, estructura, y clasificación taxonómica de las ideas, y como ayuda interna para el estudio, organización, toma de decisiones y solución de problemas (Ortega-Jiménez, 2016). A su vez, en concordancia con diferentes autores (Valdés, Menéndez \& Valdés, 2006; Kinchin, Streatfield \& Hay, 2010; MuñozGonzález \& Ontoria, 2014; Muñoz, Ariza \& Sampedro, 2015), los mapas conceptuales pueden ayudarnos a organizar, representar y almacenar nuestro conocimiento.

La creación de mapas mentales se apoya en la utilización de diferentes elementos, como pueden ser imágenes o icónicos cargados de valor semántico, códigos de colores, diferentes tipos y tamaños de letra, etc., con objeto de crear un modelo mental capaz de explicar las relaciones entre distintos niveles de información sobre un concepto o tópico (Villalustre-Martínez \& Del Moral-Pérez, 2010; Vázquez-Cano, López Meneses \& Sánchez-Serrano, 2015; Vázquez-Cano, López Meneses \& Jaén Martínez, 2017). Por su parte, Novak (2000), indica los principales elementos que componen un mapa conceptual:

- Concepto. Se entiende por concepto la palabra o término que manifiesta una regularidad en los hechos, acontecimientos ideas y/o cualidades.

- Proposición. Se establece a partir de la unión de dos o más conceptos ligados por palabras de enlace en una unidad semántica. Corresponde a la unidad principal del significado.

- Palabras de enlace. Son palabras que unen los conceptos y señalan los tipos de relación existente entre ellos.

En la imagen 1 se representa la estructura y elemento principales de un mapa conceptual multimedia (MCM). 
López Meneses, E.; Vázquez Cano, E.; Fernández Márquez, E. y López Belmonte, J.

Representaciones conceptuales multimedia de los ámbitos de actuación en educación social elaborados por estudiantes universitarios (2015-2018)

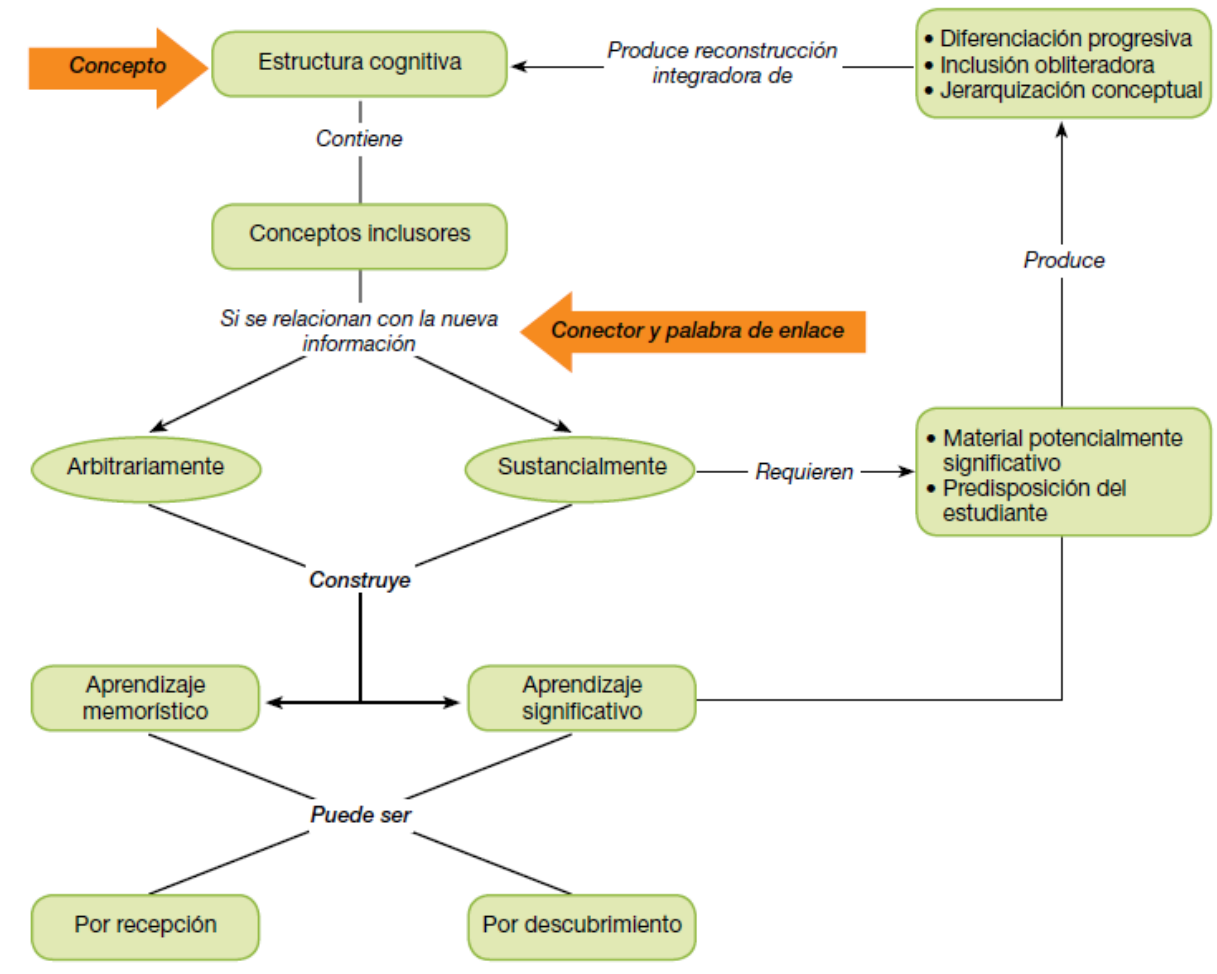

Imagen 1: Estructura y elementos de los mapas conceptuales multimedia.

Fuente: Elaboración propia a partir de Maglione y Varlotta (2011).

Asimismo, según Novak (2010), un mapa conceptual es útil en la docencia para organizar los contenidos vistos durante una unidad didáctica, es un medio de visualizar conceptos y relaciones jerárquicas entre conceptos conocidos.

\subsection{Escenario de la investigación}

La investigación describe un estudio diacrónico sobre las concepciones acerca de los ámbitos propios de la Educación Social mediante la elaboración de mapas conceptuales multimedia. El desarrollo de la misma plantea un carácter innovador y se desarrolla con 266 estudiantes pertenecientes a los cursos de Grado de Educación Social y Doble Grado de Educación Social y Trabajo Social de la Universidad Pablo de Olavide, durante los cursos académicos 2015/2016, 2016/2017 y 2017/2018.

La experiencia partía de plantear al estudiantado una reflexión introspectiva a través de mapas conceptuales multimedia (MCM) sobre los ámbitos de actuación de los profesionales en Educación Social, que debían plasmar con la aplicación Mindomo (http://www.mindomo.com), la cual permite diseñar mapas conceptuales (Mind Maps) de forma dinámica y fácil a partir de los conceptos que se le indica. Posteriormente, ellos enviaban el enlace del MCM con un breve comentario al correo enviarlas como comentarios al edublog

(http://mapasconceptualesestudiantes.blogspot.com.es/) (Imagen 2). 
López Meneses, E.; Vázquez Cano, E.; Fernández Márquez, E. y López Belmonte, J.

Representaciones conceptuales multimedia de los ámbitos de actuación en educación social elaborados por estudiantes universitarios (2015-2018)

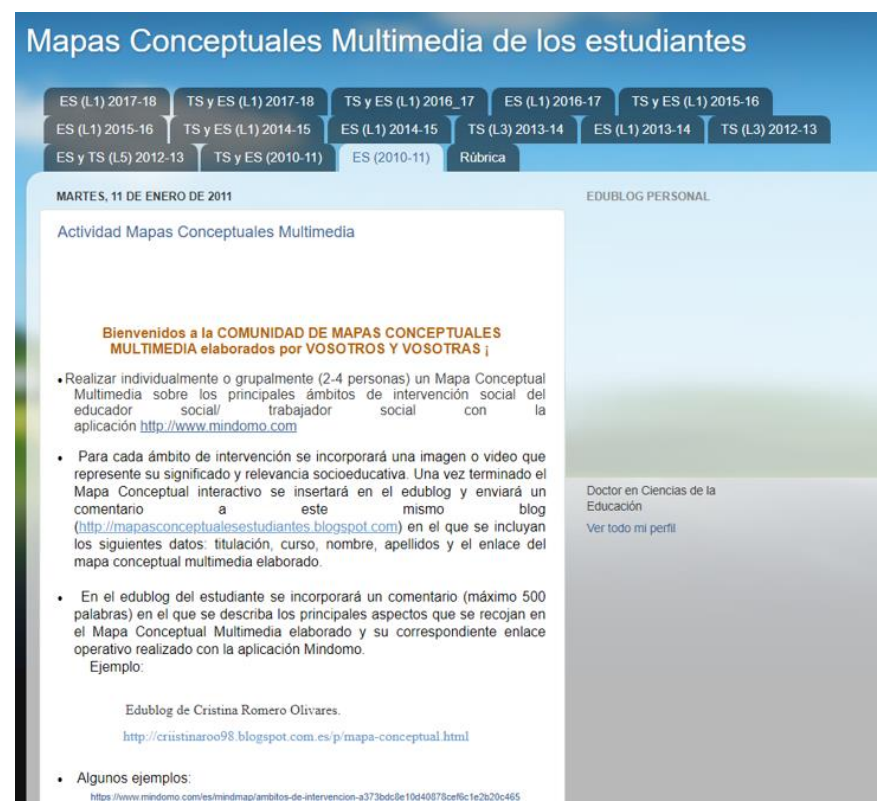

Imagen 2: Espacio virtual de la experiencia innovadora universitaria. Fuente: http://mapasconceptualesestudiantes.blogspot.com/

Por último, cada estudiante tenía que elaborar un edublog personal para la asignatura sobre las evidencias de las actividades realizadas, siendo unas de sus pestañas los Mapas Conceptuales o un título similar donde debían de añadir un comentario (máximo 500 palabras) en el que se describa tanto la estructura como los principales aspectos que se recojan en el Mapa Conceptual Multimedia elaborado y su correspondiente enlace realizado con la aplicación Mindomo (imagen 3).

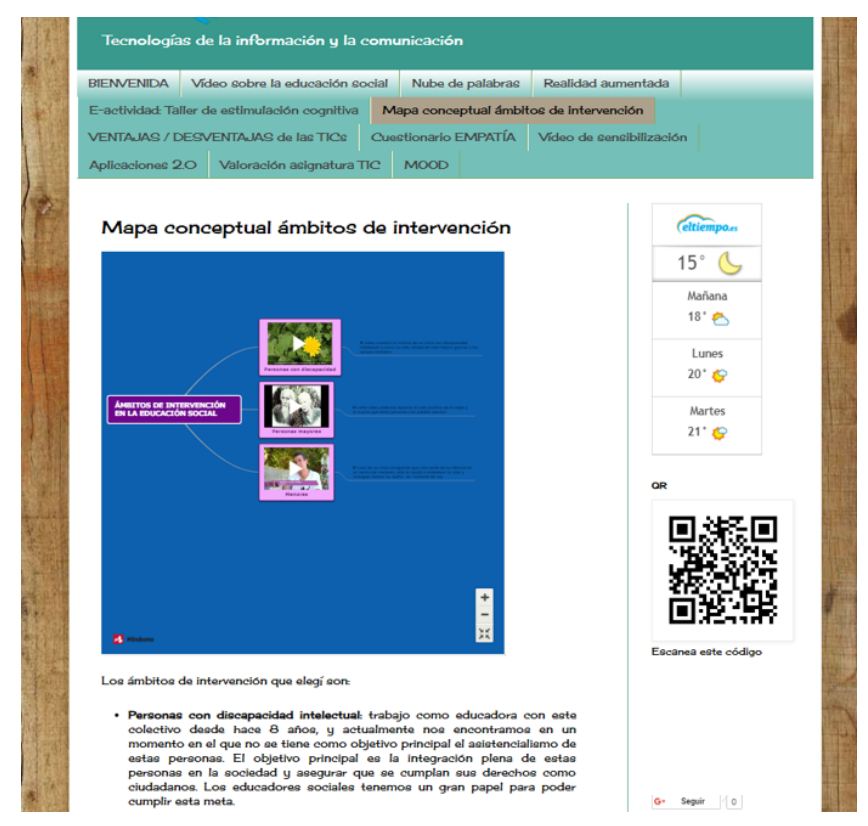

Imagen 3: Edublog de una estudiante de Educación Social del $1^{\circ}$ Curso de Grado de Educación Social. Curso académico 2017-2018.

Fuente: https://rvm3000.blogspot.com.es/ 
López Meneses, E.; Vázquez Cano, E.; Fernández Márquez, E. y López Belmonte, J.

Representaciones conceptuales multimedia de los ámbitos de actuación en educación social elaborados por estudiantes universitarios (2015-2018)

A continuación, se muestra las intenciones didácticas, el desarrollo de la investigación y los resultados más relevantes alcanzados durante el desarrollo del presente estudio.

\section{OBJETIVOS}

El presente estudio diacrónico se sustenta en las siguientes intenciones educativas.

- Investigar sobre los principales ámbitos de intervención social del futuro educador/a social seleccionado por el estudiantado del primer curso de la asignatura TIC y Educación Social de las titulaciones de Grado de Educación Social y Doble Grado de Educación Social y Trabajo Social correspondientes a los cursos académicos 2015-2016; 2016-2017 y 2017-2018.

- Analizar los estilos de representaciones gráficas que emplean los estudiantes del primer curso de las titulaciones de Grado y Doble grado de Educación Social y Trabajo Social de los tres cursos académicos (2015-2018).

\section{METODOLOGÍA}

Referente a la metodología utilizada, apuntar que ésta fue de corte cualitativo y descriptivo. Para el análisis se revisaron las 266 aportaciones realizadas por los estudiantes analizando las palabras o conjuntos de significados como unidades de registro. Posteriormente se categorizó la trama conceptual tomando como marco de referencia las pautas establecidas por diferentes autores (Bogdan \& Biklen, 1992; Miles \& Huberman, 1994):

- Fase Primera: Reducción de datos. Esta fase constituye la realización de procedimientos racionales que consisten en la categorización y codificación de los datos, identificando y diferenciando unidades de significado. Los procedimientos son:

- Categorización de los datos. La categorización implica la simplificación y selección de información para hacerla más manejable. Este proceso implica varias subfases:

- Separación de unidades. Consiste en separar segmentos de información siguiendo algún tipo de criterio como puede ser espacial, temporal, temático, gramatical.

- Identificación y clasificación de unidades. Consiste en clasificar conceptualmente las unidades que son cubiertas por un mismo tópico con significado. El procedimiento puede ser inductivo, es decir, a medida que se van examinando los datos, o deductivo, habiendo establecido previamente el sistema de categorías sobre el que se va a categorizar, tras la revisión de literatura específica sobre la temática objeto de estudio. Normalmente esta clasificación suele ser mixta.

- Síntesis y agrupamiento. Esta fase está unida realmente a la anterior dado que la propia categorización implica la síntesis. Esta fase también está presente una vez que ha concluido el 
proceso de categorización y algunas categorías se agrupan en metacategorías.

- Codificación. Es realmente la operación concreta y manipulativa por el que se asigna cada categoría a cada unidad textual. En este sentido, cada unidad seleccionada ha sido codificada para su recuento frecuencial, mediante el programa de análisis estadístico IBM SPSS Statistics 20.0, siendo el mismo "uno de los programas de mayor uso en los Estados Unidos de Norteamérica así como en América Latina [...] permite manejar bancos de datos de gran magnitud y también efectuar análisis estadísticos muy complejos" Castañeda, Cabrera, Navarro y Vries (2010), siendo de fácil utilización, y cuya implantación ha permitido cambiar los procesos de análisis estadístico en la enseñanza (Green \& Salkind, 2011). Desde un principio existieron criterios claros de distinción de unidades de registro, ya que la mayoría de los estudiantes eligieron 3 conceptos enmarcables en cada categoría.

- Fase segunda: Interpretación e inferencia. El software SPSS facilita la creación de archivos de datos de forma estructurada y organizar las bases de datos que pueden ser analizada con diversas técnicas estadísticas (imagen 4).

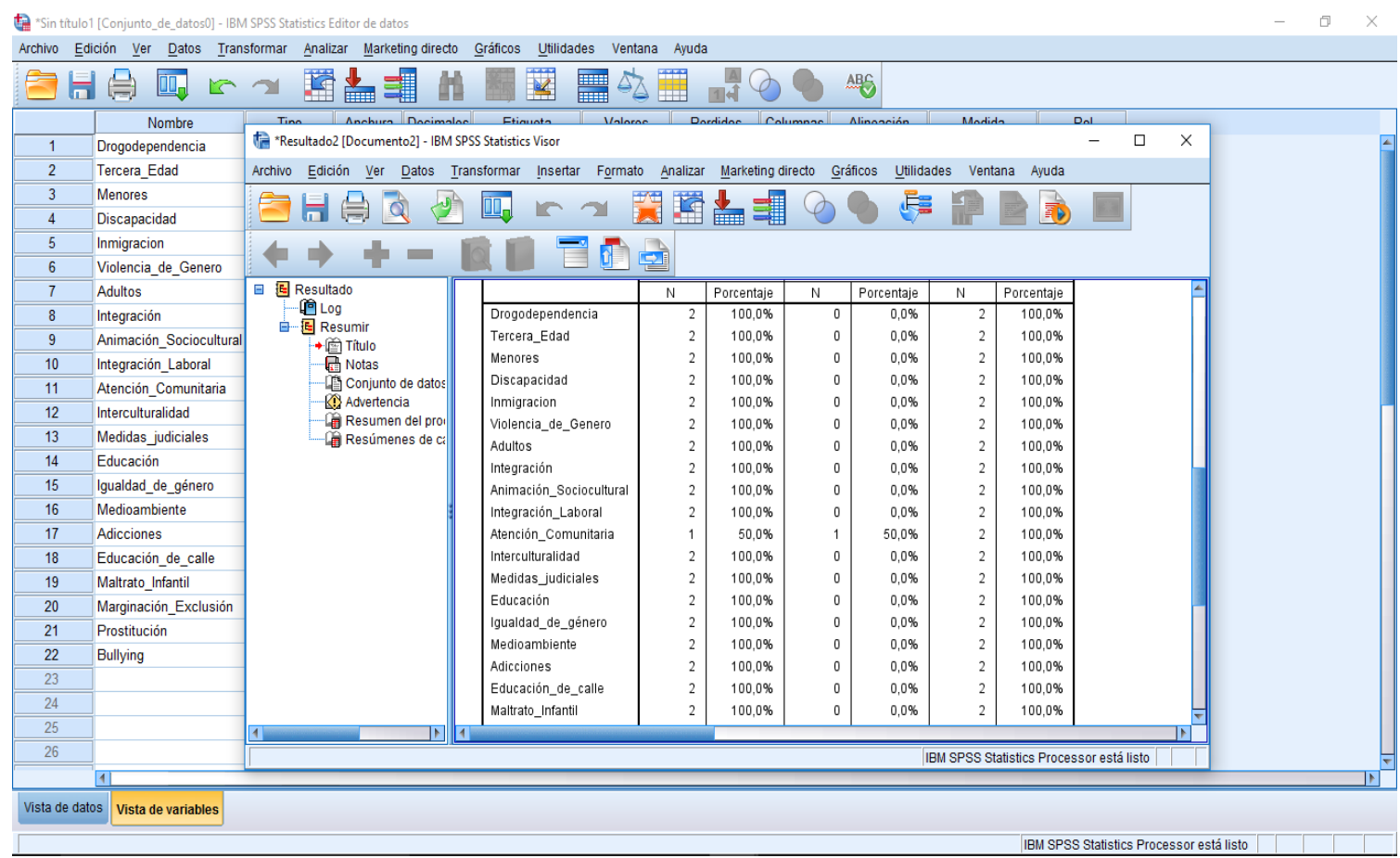

Imagen 4: Análisis categorial elaborado con IBM SPSS Statistics 20.0. Fuente: Elaboración propia.

\section{RESULTADOS}

En este apartado reflejamos los resultados obtenidos desde dos análisis diferentes: los MCM y los ámbitos de actuación en Educación Social. 
López Meneses, E.; Vázquez Cano, E.; Fernández Márquez, E. y López Belmonte, J.

Representaciones conceptuales multimedia de los ámbitos de actuación en educación social elaborados por estudiantes universitarios (2015-2018)

\subsection{MCM: Cómo los estudiantes representan sus conocimientos}

Atendiendo en primera instancia a los resultados obtenidos después de la codificación e interpretación de la información extraída de los MCM realizados por el alumnado, cabe indicar que en la forma de organización o plasmación de los contenidos por parte del alumnado destaca principal y mayoritariamente la estructuración jerarquizada, la cual prima significativamente (en torno al 70\%), sobre la estructuración en araña (30\%) como se aprecia en la gráfica 1.

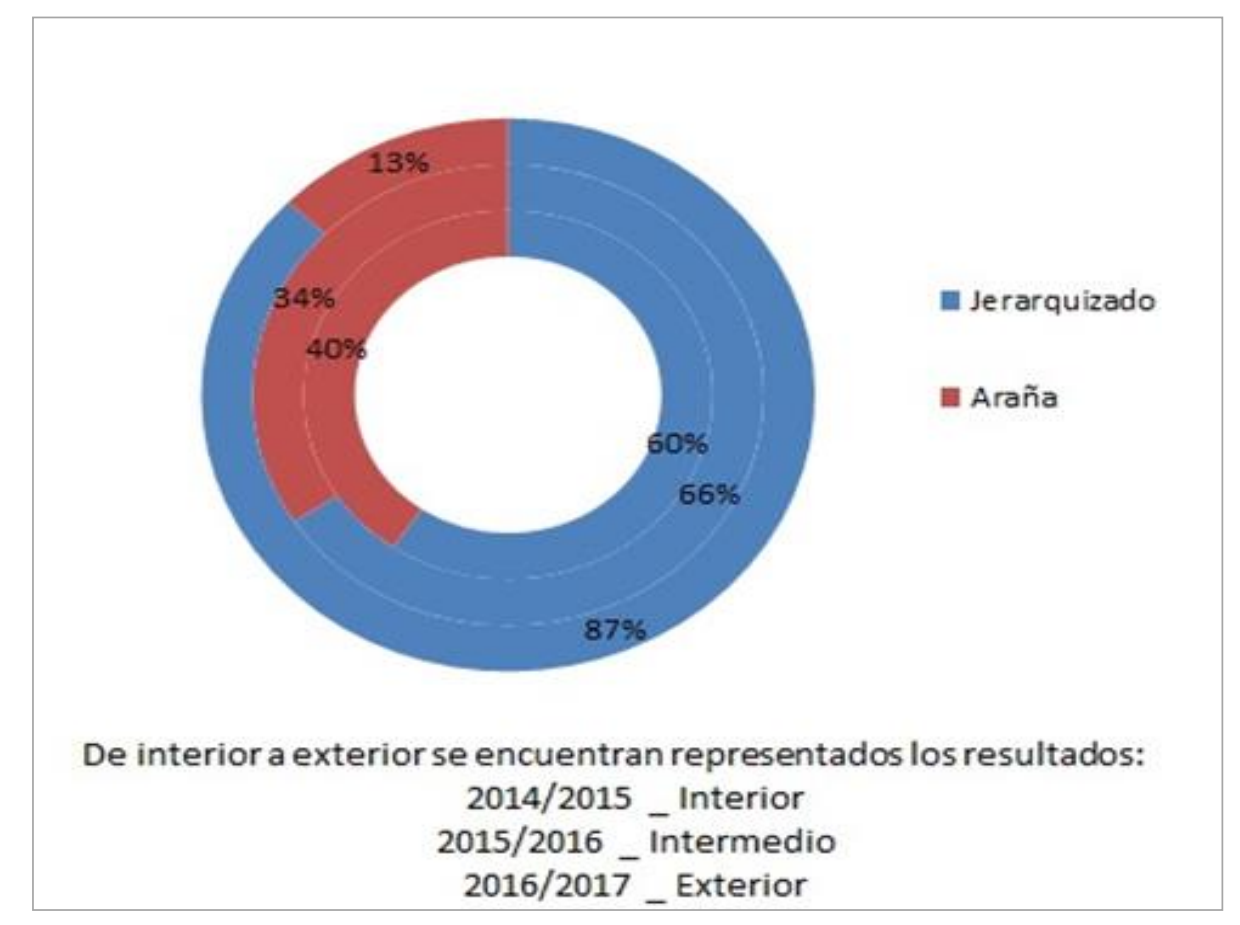

Gráfica 1: Estructuración de la representación gráfica en los MCM. Fuente: Elaboración propia.

También, es de resaltar que, durante el análisis y categorización de las aportaciones, se observa que los estudiantes que optan por la representación en araña, tampoco tienen bien integrado en qué consiste realmente los mapas conceptuales, puesto que no utilizan (en ningún caso) palabras de enlace ni conexiones entre los conceptos de niveles secundarios.

De aquellos que optan por una presentación jerarquizada, podemos observar como prevalecen las representaciones verticales, sobre la horizontalidad (60\%-40\%), encontrando una mayor diferenciación en los últimos cursos académicos, detectando un cambio de tendencia en el último curso académico (gráfica 2). 
López Meneses, E.; Vázquez Cano, E.; Fernández Márquez, E. y López Belmonte, J. Representaciones conceptuales multimedia de los ámbitos de actuación en educación social elaborados por estudiantes universitarios (2015-2018)

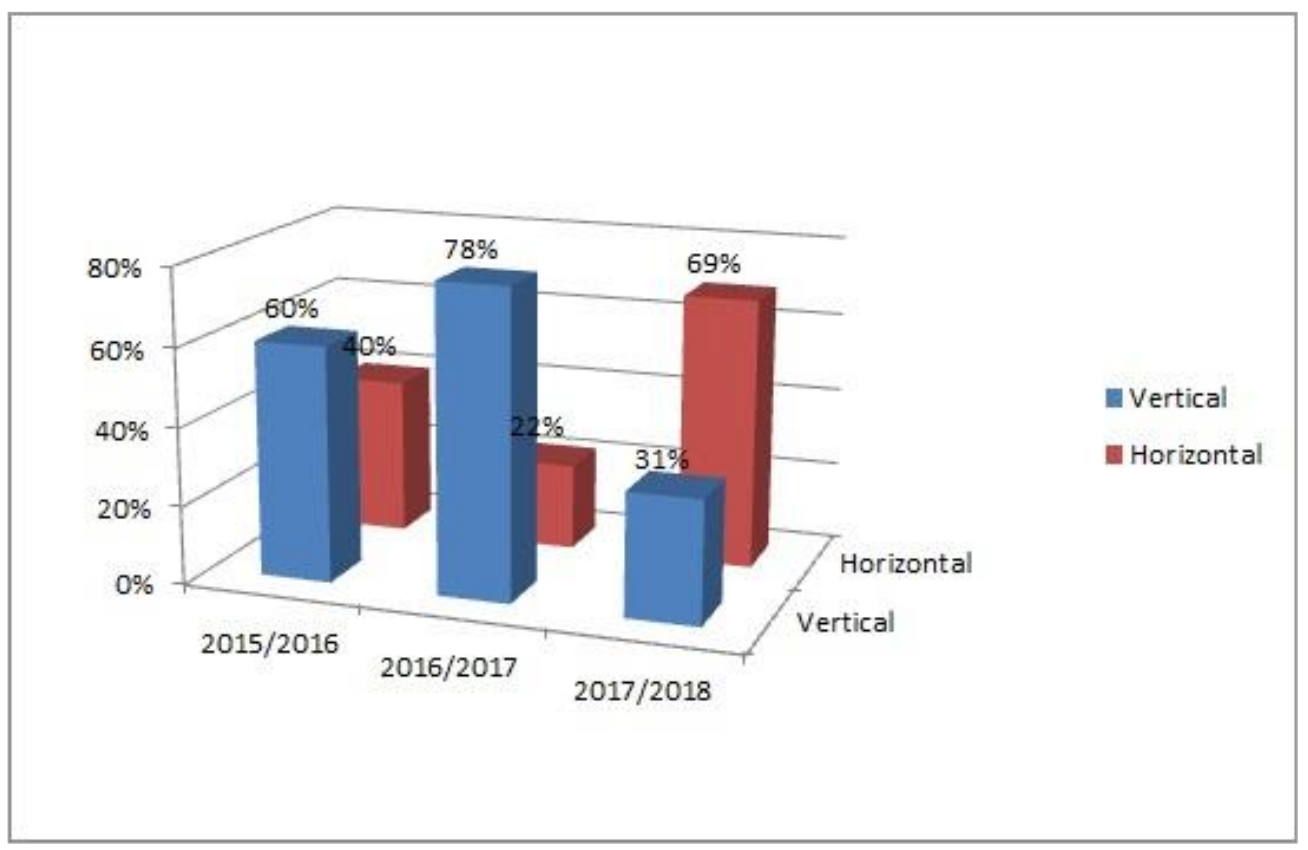

Gráfica 2: Modalidad de jerarquización de los MCM.

Fuente: Elaboración propia.

\subsection{Concepciones de los estudiantes sobre cuáles son los ámbitos de actuación en Educación Social}

Centrándonos, por otra parte, en el análisis de los ámbitos de actuación señalados por el conjunto del alumnado, cabe destacar que han establecido una media de 3,4 conceptos propios asociados a los principales ámbitos de intervención social del educador social.

Se denota, en base a sus aportaciones, una inadecuada delimitación conceptual, mezclando sectores poblacionales beneficiarios de las actuaciones (infancia, juventud, tercera edad, personas sintecho, inmigrantes...) con ámbitos de actuación (animación sociocultural, drogodependencia, violencia de género, prostitución, prisión, atención sociocomunitaria...).

Al margen de este aspecto, obtenemos la siguiente tabla de frecuencias de respuestas (tabla 1 ):

Tabla 1. Frecuencia de respuestas sobre los ámbitos de intervención en Educación Social.

\begin{tabular}{|c|c|c|c|}
\hline $\begin{array}{c}\text { Ámbitos intervención } \\
\text { Educador Social }\end{array}$ & $\begin{array}{c}\text { Frecuencia de } \\
\text { Respuesta }\end{array}$ & $\begin{array}{c}\text { Ámbitos intervención } \\
\text { Educador Social }\end{array}$ & $\begin{array}{c}\text { Frecuencia de } \\
\text { Respuesta }\end{array}$ \\
\hline Adicciones (a tecnologías) & 35 & Infancia & 85 \\
\hline Adolescencia & 39 & Inmigración & 75 \\
\hline Adultos & 101 & Integración & 23 \\
\hline Alcohol en Menores & 11 & Integración laboral & 26 \\
\hline
\end{tabular}


López Meneses, E.; Vázquez Cano, E.; Fernández Márquez, E. y López Belmonte, J. Representaciones conceptuales multimedia de los ámbitos de actuación en educación social elaborados por estudiantes universitarios (2015-2018)

\begin{tabular}{|c|c|c|c|}
\hline Animación Sociocultural & 68 & Interculturalidad & 8 \\
\hline Atención Comunitaria & 26 & Mediación Familiar & 7 \\
\hline Discapacidad / Salud Mental & 138 & Medidas judiciales & 23 \\
\hline Discriminación por genero & 5 & Medioambiente & 10 \\
\hline Drogodependencia & 132 & Pobreza & 1 \\
\hline Educación & 16 & Prostitución & 5 \\
\hline Educación de calle & 13 & Racismo & 5 \\
\hline Exclusión Social & 22 & Salud & 5 \\
\hline Explotación infantil & 2 & Sin techo / Personas sin hogar & 13 \\
\hline Formación / Asesoramiento & 5 & Tercera Edad & 126 \\
\hline Genero & 6 & Violencia de Genero & 55 \\
\hline Homofobia & 2 & Violencia Infantil (Maltrato, & 10 \\
\hline Igualdad de género & 4 & Bullying) & \\
\hline
\end{tabular}

Fuente: Elaboración propia.

Analizando los porcentajes, mostramos en la gráfica 3 los más destacables, siendo éstos: personas con discapacidad y salud mental (15,37\%), drogodependencia $(14,70 \%)$, tercera edad $(14,03 \%)$, adultos $(11,25 \%)$, infancia $(9,47 \%)$, inmigración $(8,35 \%)$, animación sociocultural $(7,57 \%)$, violencia de género $(6,12 \%)$ y adolescencia (4,34\%).

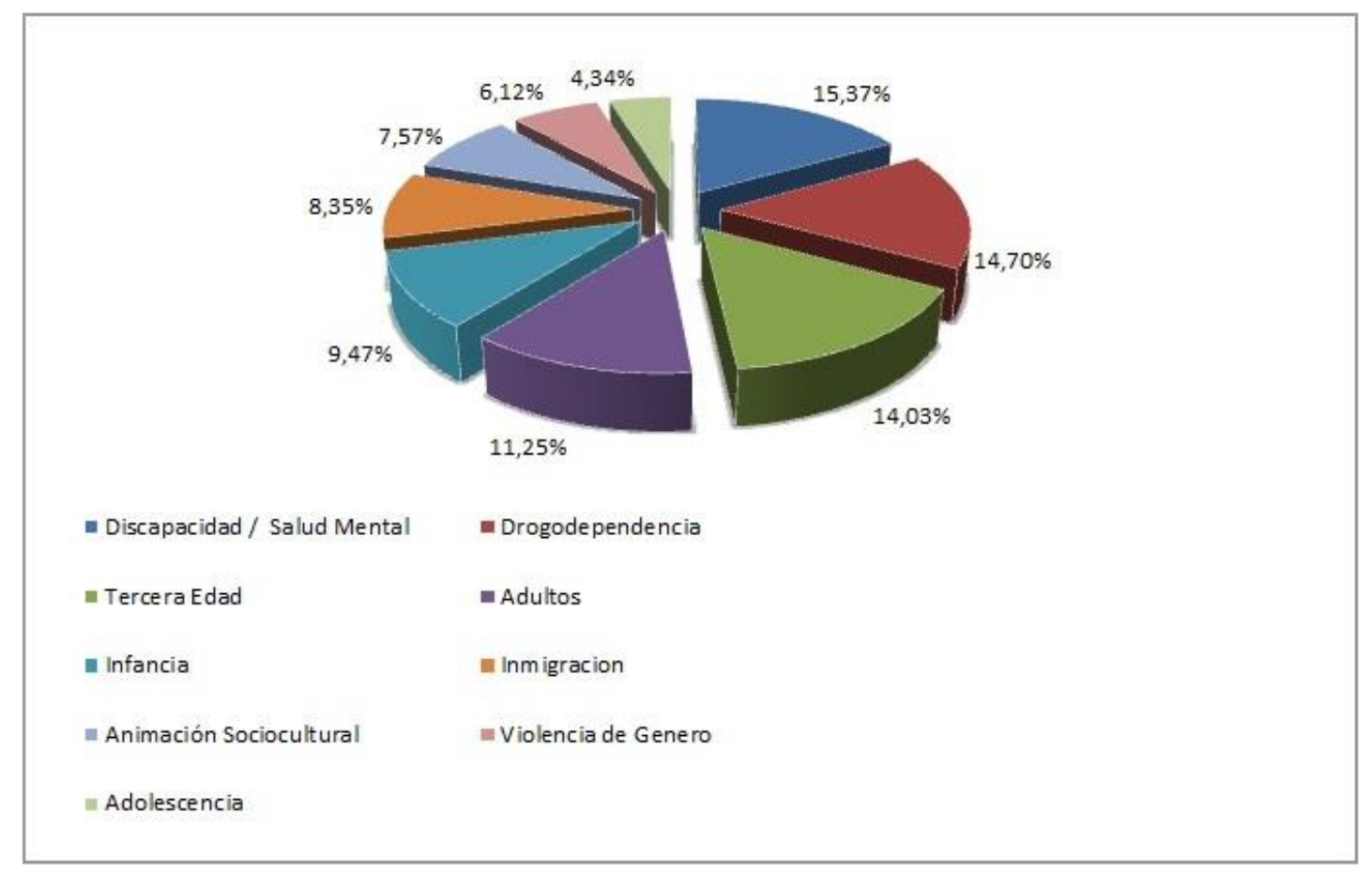

Gráfica 3: Principales ámbitos de actuación en Educación Social.

Fuente: Elaboración propia. 
López Meneses, E.; Vázquez Cano, E.; Fernández Márquez, E. y López Belmonte, J.

Representaciones conceptuales multimedia de los ámbitos de actuación en educación social elaborados por estudiantes universitarios (2015-2018)

Atendiendo al análisis diacrónico entre los cursos escolares 2015/2016, 2016/2017 y 2017/2018, (gráfica 4), se infiere una progresión y aparición de nuevos conceptos entre las concepciones o cómo otros aspectos han dejado de tener importancia.

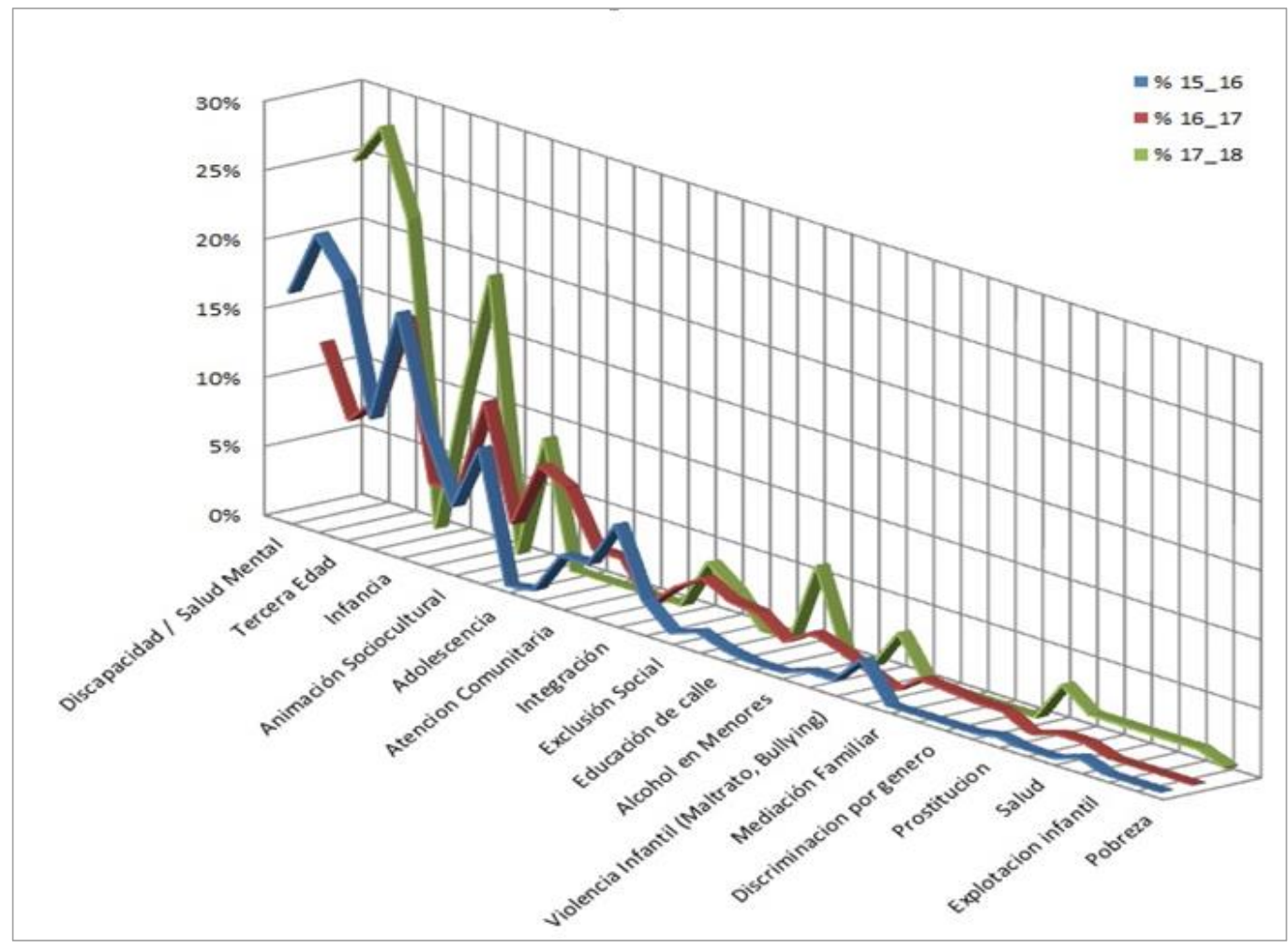

Gráfica 4: Comparativa de los ámbitos de intervención de la Educación Social. Análisis diacrónico.

Fuente: Elaboración propia.

\section{DISCUSIÓN Y CONCLUSIONES}

En una primera instancia, se infiere en concordancia con Reyes-Santander, P. A. y Ramos-Rodríguez (2018) que la generación de mapas presenta dificultades iniciales para los estudiantes y es aún más complejo, cuando se les invitan a que lo expresen y lo comuniquen. No obstante, a lo largo de la experiencia se corrobora con Valverde-Berrocoso y Garrido-Arroyo (2002) que este tipo de técnica didáctica de aprendizaje visual ayudan a los estudiantes en el proceso de adquisición y retención de nueva información estableciendo conexiones entre el conocimiento nuevo y el ya existente.

Con respecto a los ámbitos de intervención del presente estudio, son similares a otros estudios de investigación (Kearney et al. 2000; British Association of Social Workers, 2002; Cunningham, 2004): los servicios sociales, la violencia de género, la inmigración o las personas sin hogar.

También, es de resaltar de esta experiencia utilizando edublog y MCM, como apuntan en otros trabajos autores como O'Donnell (2006), Farmer, Yue y Brooks 
López Meneses, E.; Vázquez Cano, E.; Fernández Márquez, E. y López Belmonte, J.

Representaciones conceptuales multimedia de los ámbitos de actuación en educación social elaborados por estudiantes universitarios (2015-2018)

(2008), Cabero, Ballesteros y López-Meneses (2015), la buena valoración que nuestros estudiantes realizan del software Mindomo y de los blogs, como aplicaciones de fácil manejo, colaborativas, intuitivas y muy útiles con la posibilidad de agregar imágenes, comentarios, enlaces, gráficos y videos de todo tipo y difundirlo a través de Internet. Es decir, permiten corroborar cómo la utilización de aplicaciones relacionadas con el software social constituye una práctica adecuada y útil para que los estudiantes puedan desempeñar un papel activo en su proceso formativo y pongan en juego habilidades de aprendizaje de orden superior.

Por último, en la misma línea discursiva de Pontes (2014) hay que destacar el hecho de que este tipo de mapas, empleados en estos tipos de actividades formativas, obligan a reflexionar sobre el propio conocimiento a quienes los realizan y ayudan a visualizar las deficiencias del proceso de aprendizaje de cualquier materia en un momento dado.

Con referencia a las líneas de futuro se constata que estudios didácticos de este tipo permiten explorar, razonar y analizar los contenidos curriculares de las asignaturas y, a su vez, son instrumentos metodológicos metacognitivos óptimos para el enriquecimiento competencial del educando. En esta línea investigadora, actualmente, desde el grupo de investigación Eduinnovagogía ${ }^{\circledR}$ (HUM-971) U.R.L http://bit.ly/1sGHwqO estamos estudiando su viabilidad para desarrollarlo en otras instituciones educativas latinoamericanas.

\section{REFERENCIAS}

Aguaded, J. I.; López-Meneses, E. y Jaén, A. (2013). Portafolios electrónicos universitarios para una nueva metodología de enseñanza superior. Desarrollo de un material educativo multimedia (MEM). Revista de Universidad y Sociedad del Conocimiento (RUSC), 10(1), 7-28. Recuperado de http://journals.uoc.edu/ojs/index.php/rusc/article/view/v10n1-aguaded-lopezjaen/v10n1-aguaded-lopez-jaen-es

Bogdan, R. C. \& Biklen, S. K. (1992). Qualitative research for education: An introduction to theory and methods (2nd Ed.). Boston, MA: Allyn and Bacon.

British Association of Social Workers (2002). The Code of Ethics for Social Work. Birmingham: British Association of Social Workers.

Cabero, J.; Ballesteros, C. y López-Meneses, E. (2015). Los mapas conceptuales interactivos como recursos didácticos en el ámbito universitario. Revista Complutense de Educación, (26), 51-76. doi: http://dx.doi.org/10.5209/rev RCED.2015.v26.43815

Cunningham, S. (2004). Children, Social Policy and the State, in Lavalette, M. \& Pratt, A. (Eds.), Social Policy: A Conceptual and Methodological Introduction. Sage: London. 
López Meneses, E.; Vázquez Cano, E.; Fernández Márquez, E. y López Belmonte, J. Representaciones conceptuales multimedia de los ámbitos de actuación en educación social elaborados por estudiantes universitarios (2015-2018)

Farmer, B.; Yue, A. \& Brooks, C. (2008). Using blogging for higher order learning in large cohort university teaching: A case study. Australasian Journal of Educational Technology, 24(2), 123-136.

Fernández-Márquez, E.; Vázquez-Cano, E. y López Meneses, E. (2016). Los mapas conceptuales multimedia en la educación universitaria: recursos para el aprendizaje significativo. Campus Virtuales 5(1), 10-18.

Kearney, P.; Levin, E. \& Rosen, G. (2000). Alcohol, Drugs and Mental Health Problems: Working with Families. London: National Institute for Social Work.

Kinchin, I. M.; Streatfield, D. \& Hay, D. (2010). Using concept mapping to enhance the research interview. International Institute for Qualitative Methodology, $9(1)$, 52-68.

López Meneses, E.; Vázquez-Cano, E. y Jaén Martínez, A. (2017). Los portafolios digitales grupales, una estrategia metodológica para mejorar el proceso de enseñanza-aprendizaje en la Universidad: un estudio diacrónico en la Universidad Pablo de Olavide (2009-2015). Revista de Humanidades, (31). doi: http://dx.doi.org/10.5944/rdh.31.2017.19076

Maglione, C. y Varlotta, N. (2011). Mapas conceptuales digitales. Buenos Aires: Educar S. E.

Miles, M. B. \& Huberman, A. (1994). Qualitative data analysis: an expanded sourcebook. Newbury Park, CA: Sage.

Muñoz, J. M.; Ariza, C. y Sampedro, B. E. (2015). La aplicación de los mapas mentales en Educación Primaria. International Journal of Educational Research and Innovation (IJERI), 4, 70-89.

Muñoz-González, J. M. y Ontoria, A. (2014). El uso de mapas mentales en la formación inicial docente. Revista Latinoamericana de Tecnología Educativa, RELATEC, 13(2), 77-88.

Novak, J. (2000). The Theory Underlying Concept Maps and How To Construct Them. Recuperado de http://stanford.io/2xJcJQd

Novak, J. D. \& Cañas, A. J. (2008). The Theory Underlying Concept Maps and How to Construct Them. Technical Report IHMC CmapTools 2006-01 Rev 01-2008. Florida Institute for Human and Machine Cognition. Recuperado de http://cmap.ihmc.us/Publications/ResearchPapers/TheoryUnderlyingConceptMaps.pdf

Novak, J. D. (2010). Learning, creating, and using knowledge: Concept maps as facilitative tools in schools and corporations. Nueva York: Routledge. 
López Meneses, E.; Vázquez Cano, E.; Fernández Márquez, E. y López Belmonte, J. Representaciones conceptuales multimedia de los ámbitos de actuación en educación social elaborados por estudiantes universitarios (2015-2018)

O'Donnell, M. (2006). Blogging as pedagogic practice: Artefact and ecology. Asia Pacific Media Educator, 17, 5-19.

Ortega-Jiménez, A. (2016). Los mapas conceptuales como método de enseñanza en derecho internacional privado. Actualidad Jurídica Iberoamericana, núm. 4.bis (extraordinario), 232-245.

Pontes, A. (2014). Representación del conocimiento físico del alumnado universitario mediante mapas conceptuales elaborados con CmapTools. Alambique: Didáctica de las Ciencias Experimentales, 76, 34-42.

Prieto, A. B. \& Chrobak, R. (2013). Integración de TICs, investigación y herramientas metacognitivas en la educación de ciencias y ambiental. Estudio de caso: disponibilidad de agua de las cuencas del noroeste de Patagonia y su relación con la actividad solar. Journal for Educators, Teachers and Trainers, 4(1), 132-141.

Reyes-Santander, P. A. y Ramos-Rodríguez, E. (2018). Mapas conceptuales en educación matemática a nivel universitario. Revista Electrónica de Investigación Educativa, 20(2), 25-36. doi: https://doi.org/10.24320/redie.2018.20.2.1657

Rodríguez-García, Y. M. (2017). Reconceptualizacion de la educación en la era digital: educomunicacion, redes de aprendizaje y cerebro. Factores claves en los actuales escenarios de construcción de conocimiento. Revista de Comunicación de la SEECI, (42), 85-118. doi: https://doi.org/10.15198/seeci.2017.42.85-118

Valdés, M. A., Menéndez, L. M. y Valdés, V. G. (2006). Los mapas conceptuales: un recurso para el aprendizaje apoyado en tecnologías. Píxel-Bit. Revista de Medios y Educación, 28, 89-97.

Valverde-Berrocoso, J. y Garrido-Arroyo, M.a C. (2002). El mapa conceptual: software de diseño y creación. Extremadura: Publicaciones Extremadura.

Vázquez-Cano, E.; López Meneses, E. \& Jaén Martínez, A. (2017). The group e-portfolio to improve Teaching-Learning process at University. Journal of e-Learning and Knowledge Society, 13(2), 65-76. doi: https://doi.org/10.20368/1971-8829/1221

Vázquez-Cano, E.; López Meneses, E. \& Sánchez-Serrano, J. L. (2015). Analysis of social worker and educator's areas of intervention through multimedia concept maps and online discussion forums in Higher Education. Electronic Journal of eLearning, 13(5), 333-346.

Villalustre-Martínez, L. y Del Moral-Pérez, E. (2010). Mapas conceptuales, mapas mentales y líneas temporales: objetos "de" aprendizaje y "para" el aprendizaje en Ruralnet. Revista Latinoamericana de Tecnología Educativa RELATEC, 9(1), 15-27. 
López Meneses, E.; Vázquez Cano, E.; Fernández Márquez, E. y López Belmonte, J.

Representaciones conceptuales multimedia de los ámbitos de actuación en educación social elaborados por estudiantes universitarios (2015-2018)

\section{AUTORES:}

\section{Eloy López Meneses}

Profesor Titular de Universidad en el Departamento de Educación y Psicología Social de la Universidad Pablo de Olavide (Sevilla, España). Doctor en Ciencias de la Educación y Premio extraordinario de tesis doctoral por la Universidad de Sevilla. Especializado en MOOC, TIC y Diseño didáctico MEM. Director del Grupo de Investigación Eduinnovagogía (HUM-971). Editor de la Revista Internacional: "International Journal of Educational Research and Innovation" (IJERI). Líneas de investigación: MOOC universitario, Realidad Aumentada/ Virtual, TIC en contextos formativos, diseño e implementación de Materiales Educativos Multimedia (MEM) y formación de educadores/as en tecnologías emergentes.

elopmen@upo.es

Orcid ID: http://orcid.org/0000-0003-0741-5367

\section{Esteban Vázquez-Cano}

Profesor de la Facultad de Educación en el Departamento de Didáctica, Organización Escolar y Didácticas Especiales. Doctor en Ciencias de la Educación con Premio Extraordinario. En la actualidad dirige el Máster de Inspección y Supervisión Educativa en la UNED y sus líneas de investigación prioritarias son la Organización y Supervisión escolar, el Aprendizaje móvil-ubicuo, el Lenguaje Digital y los Cursos Online Masivos en Abierto (MOOC), temáticas sobre las que ha publicado más de 200 contribuciones científicas (Google Scholar, Scopus).

evazquez@edu.uned.es

Orcid ID: https://orcid.org/0000-0002-6694-7948

\section{Esther Fernández Márquez}

Profesora de la Universidad Pablo de Olavide. Doctora en Educación y Comunicación en la Universidad de Málaga. Miembro del grupo de investigación Eduinnovagogía (HUM-971). Línea de investigación: Formación del profesorado en competencias digitales e innovación en tecnologías emergentes.

efermar2@upo.es

Orcid ID: https://orcid.org/0000-0003-0111-2533

\section{Jesús López Belmonte}

Doctor en Ciencias de la Educación por la Universidad de Granada (España). Posee un Máster universitario oficial en Tecnología Educativa y Competencias Digitales por la Universidad Internacional de la Rioja. Ejerce su labor docente en la Universidad Internacional de Valencia, dentro del Máster de Secundaria bajo la dirección de TFM y en el Centro Concertado Beatriz de Silva, en la Ciudad Autónoma de Ceuta (España). Desarrolla su labor investigadora en el Grupo de Investigación AREA HUM672 (Análisis de la Realidad EducativA).

jesus.lopezb@campusviu.es

Orcid ID: https://orcid.org/0000-0003-0823-3370 